\title{
Diseño de un protocolo de pruebas del sistema airbag mediante la interpretación de oscilogramas de operación
}

\section{Design of an airbag system test protocol through the interpretation of operation oscillograms}

Luis Xavier Orbea Hinojosa

Ing. Gualotuña Quishpe Elvis Patricio

José Antonio Toapaxi Csanoba

José Adrian Rodriguez Rodriguez

Universidad Tecnológica Equinoccial, Ecuador

Ing. Daniela Alexandra Jeréz Mayorga

Universidad Internacional del Ecuador, Ecuador

Autor para correspondencia: luis.orbea@ute.edu.ec,elvis.gualotuna@ute.edu.ec, tcja7012823@ute.edu.ec, jose.rodriguez@ute.edu.ec,djerez@internacional.edu.ec Fecha de recepción: 02 de Junio de 2017 - Fecha de aceptación: 15 de Septiembre de 2017

Resumen: En el estudio del sistema AIRBAG de un vehículo de turismo 1600cc; se enfocaron y analizaron sus diagramas en función de los elementos del sistema [1], se tomaron datos de los sensores de velocidad, sensores de impacto, módulo de control, cableado del sistema, espiral contactor, socket de conexiones; además el presente proyecto utilizó equipos de medición y diagnóstico, multímetro automotriz, scanner, y osciloscopio. Se creó las conexiones entre los instrumentos de medición y de diagnóstico adecuando el vehículo para pruebas experimentales y de esta manera obtener datos de los elementos del sistema. Se tomaron datos específicos de cada elemento utilizando flujo gramas de operación, los datos fueron: señales eléctricas de operación normal, códigos de fallas del sistema; y mediante un osciloscopio se obtuvo oscilogramas de operación de cada elemento, se interpretó y comparó estos datos con los esperados por el fabricante para desarrollar cuadros de desviación, PIDs y las soluciones propuestas. Los datos obtenidos en condiciones normales de operación y de falla fueron a base de oscilogramas de operación, una vez evaluados estos datos, se diseñaron los protocolos de pruebas que nos permitieron realizar un trabajo de mantenimiento y control de funcionamiento más eficiente.

Palabras Clave: airbag; seguridad pasiva; oscilogramas; protocolo de pruebas

Abstract: In the study of the system AIRBAG of a vehicle of tourism 1600cc; they focused and analyzed his graphs depending on the elements of the system [1], there took information of the speed sensors, sensors of impact, module of control, wired up of the system, spiral contactor, socket of connections; in addition the present project used equipments of measurement and diagnosis, multimeter self-propellingly, scanner, and oscilloscope. The connections were created between the instruments of measurement and of diagnosis adapting the vehicle for experimental tests and hereby to obtain information of the elements of the system. There took specific information of every element using flow grasses of operation, the information was: electrical signs of normal operation, codes of faults of the system; and by means of an oscilloscope there were 
obtained oscillograms of operation of every element, this information was interpreted and compared with waited by the manufacturer to develop pictures of diversion, PIDs and the proposed solutions. The information obtained in normal conditions of operation and of fault was based on oscillograms of operation, once evaluated this information, there were designed the protocols of tests that allowed us to realize a work of maintenance and control of more efficient functioning.

Key words: airbag; passive safety; oscillograms; protocol of tests

\section{Introducción}

Sus inicios fueron sobre la base de una norma de seguridad que los norteamericanos dictaron en el año 1967 por la Federal Motor Vehicule Safety Standard, esta norma provoca a que algunos de los fabricantes de vehículos empiecen a desarrollar nuevos sistemas de seguridad en los habitáculos, como el airbag [8], y desde esa época se ha venido desarrollando este sistema de seguridad.

El AIRBAG es un sistema de seguridad pasiva y está diseñado para reducir el riesgo de lesiones, principalmente en la cabeza y la parte superior del tronco, en conductores y ocupantes o pasajeros. El sistema Airbag es comandado por la unidad electrónica para su activación, esto lo realiza en base a las señales recibidas por el sensor del sistema que está ubicado estratégicamente para detectar paradas bruscas por causa de una colisión, esta señal eléctrica que recibe la unidad de control, es a su vez consiste en una bolsa que se expande como resultado de la liberación de aire provocado por la activación de los detonantes y la generación química entre las pastillas de nitrógeno; aunque en la actualidad se utiliza aire comprimido pero su objetivo se mantiene en caso de colisión fortuita (deceleración) del vehículo para evitar ser arrojados al conductor o a sus acompañantes y evitar que se proyecten contra el parabrisas o la columna de la dirección y otras partes duras de la estructura del vehículo causa de gran parte de las lesiones, sin embargo, esto no constituye una promesa de que ante siniestros de elevada magnitud de los ocupantes resulten sin ningún tipo de lesión en caso de accidente[7], y asegura entre un 40 y 50\% de esperanza de vida su utilización es un complemento del cinturón de seguridad mas no un remplazo porque si bien se cita con anterioridad que evita salir disparados por la desaceleración del vehículo la activación de airbag sin tener un cinturón de seguridad el conductor o los ocupantes puede traer resultados pocos favorables por la presión a la que se infla el airbag que aproximadamente es 250-300 bares de presión.

El airbag entra en acción ante un cambio brusco de la inercia general del automóvil, como sucede en caso de un impacto frontal contra un obstáculo u otro vehículo. El llenado de la bolsa se produce mediante una detonación pirotécnica, de modo que se forma un cojín amortiguador entre el conductor o pasajero y los elementos rígidos que tiene delante, amortiguando e incluso evitando el impacto.

En dependencia de la tecnología y el énfasis que aplican las distintas firmas automotrices (marcas) respecto a la seguridad pasiva que se le pueda ofrecer a sus compradores va derivar en una mayor esperanza de vida en caso de accidentes ocasionados como no ocasionados (fortuitos). 


\section{- Eficacia}

En un estudio de la Administración de Tráfico Norteamericana (la NHTSA), se cifraba la eficacia del airbag para reducir muertes en caso de accidente, en un $13 \%$ cuando no se llevaba además puesto el cinturón de seguridad, la eficacia de cinturón de seguridad solo en un $45 \%$ y la eficacia combinada del cinturón de seguridad y de airbag en el 50\% en la reducción de la mortalidad por accidentes de tráfico. Si analizamos la eficacia en la reducción de lesiones según las distintas zonas del cuerpo, los datos son más notables aún, ya que para la cabeza el riesgo de lesión grave se reduce hasta en un $83 \%$ cuando se usan el cinturón de seguridad y el airbag juntos. [9]

Tabla 1: Eficacia del airbag con y sin el cinturón en porcentaje.

Eficiencia estimada de los sistemas de protección del ocupante en la reducción del riesgo de muerte para los conductores de turismo.

\begin{tabular}{ll} 
Sistema usado & Reducción de mortandad \\
Airbag + cinturón & $50 \%$ \\
Cinturón solo & $45 \%$ \\
Airbag solo & $13 \%$ \\
\hline
\end{tabular}

Algunos modelos de automóviles disponen también de dispositivos laterales de este tipo, para proteger a los ocupantes del vehículo en caso de colisión lateral y los automóviles más potentes vienen instalando los airbags de cortina de techo, para la protección de los impactos laterales de la cabeza.

Según la empresa Volkswagen [2], que tiene cierta ventaja a las otros firmas automotrices en temas de seguridad se adjudica ser uno de los pioneros en la creación de dos tipos de AIRBAG frontales para diferenciar dos continentes de acuerdo a índices estadísticos como: peso, hábitos (uso del cinturón de seguridad), forma de conducir e índice de accidentes, entre otros, los dos tipos son: EUROPEO y AMERICANO; las principales diferencias radica en su volumen la velocidad de accionamiento y dimensiones, ya que en europeos su volumen varían entre 30 a 45 libras para el conductor y para el acompañante va de 70 a 90 libras, en cambio en el americano para conductores va de 60 a 80 libras y acompañes va de 130 a 150 libras acompaña de una velocidad más baja y mayor dimensión que el europeo.

\section{Materiales y Métodos}

Se pretende mostrar los componentes del AIRBAG su método de accionamiento y el aporte que ha dado el sistema para reducir la tasa de mortalidad desde su inicio hasta la actualidad.

Proyectar influencia positiva que tienen el sistema airbag mediante pruebas técnicas y los aspectos negativos en su no accionamiento si sucede una colisión.

El airbag debe cumplir todos los requisitos de un producto de consumo, en este caso de automoción; es decir, no debe tener posibilidad de fallos que causen lesiones y debe soportar las condiciones de uso de un automóvil (vibraciones, temperatura, humedad...) durante toda la vida del vehículo. 


\section{Componentes del sistema airbag}

La unidad de control. - Se encarga directamente del disparo del sistema, para lo cual debe disponer de un sistema de seguridad que impida disparos accidentales. Básicamente, este sistema dispone de dos sensores, que habitualmente se encuentran dentro de la propia centralita.

El cojín inflable. - Este es uno de los elementos más importantes y a la vez más peligroso del sistema, ya que en su interior se encuentra el material explosivo capaz de inflar el cojín en milésimas de segundo. Su composición física es de un tejido de poliamida, sin recubrimiento. Tiene varias aberturas de escape en la parte trasera para permitir la salida uniforme del gas al ser comprimida por el tórax y la cabeza. El cojín de aire para el conductor va alojado en el volante de la dirección y se infla cuando se produce una colisión a más de $30 \mathrm{~km} / \mathrm{h}$. [6]

El sensor de deceleración. - Va integrado en la unidad de control como un elemento electrónico más. Se trata de un sensor piezoeléctrico capaz de convertir la deceleración mecánica en señal eléctrica (al modificar la presión aplicada al sensor, la tensión de salida cambia).

Así, en un impacto con fuerte deceleración, la inercia que actúa sobre el sensor modifica la señal de salida, indicando a la central, con toda precisión, la velocidad del impacto y deceleración que se provocará. Estos datos se consultan a la memoria de la ECU y, si se considera necesaria la intervención del Airbag, se provoca el encendido de los detonadores situados en el cojín o cojines hinchables.

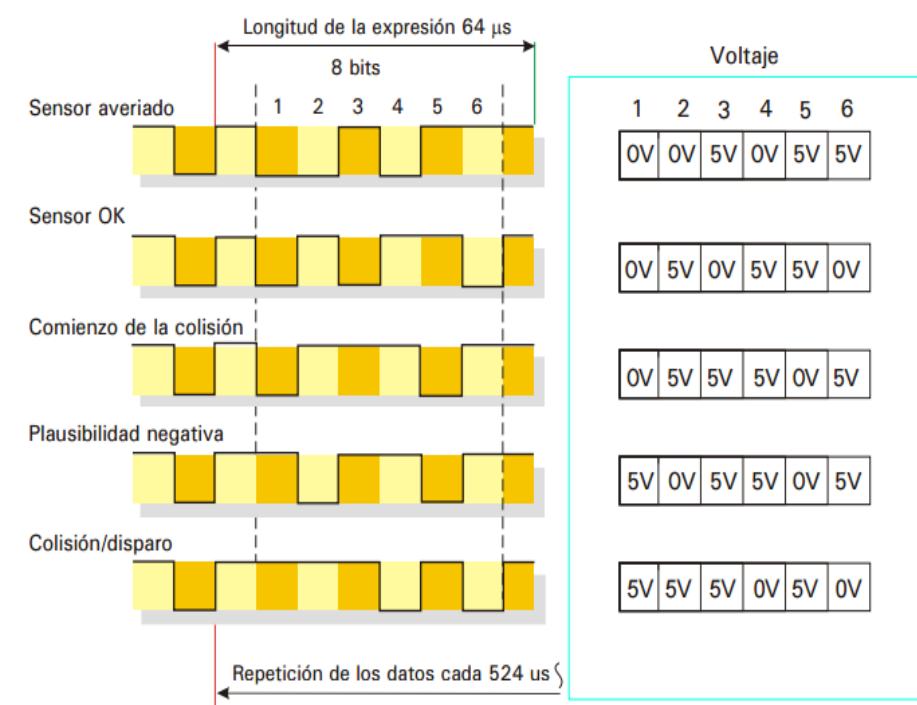

Figura 1: Frecuencia con la que el sensor envía la información al módulo de control. La repetición de los datos enviados se realiza cada 524 microsegundos

Fuente: Volkswagen; elementos electrónicos de seguridad. 


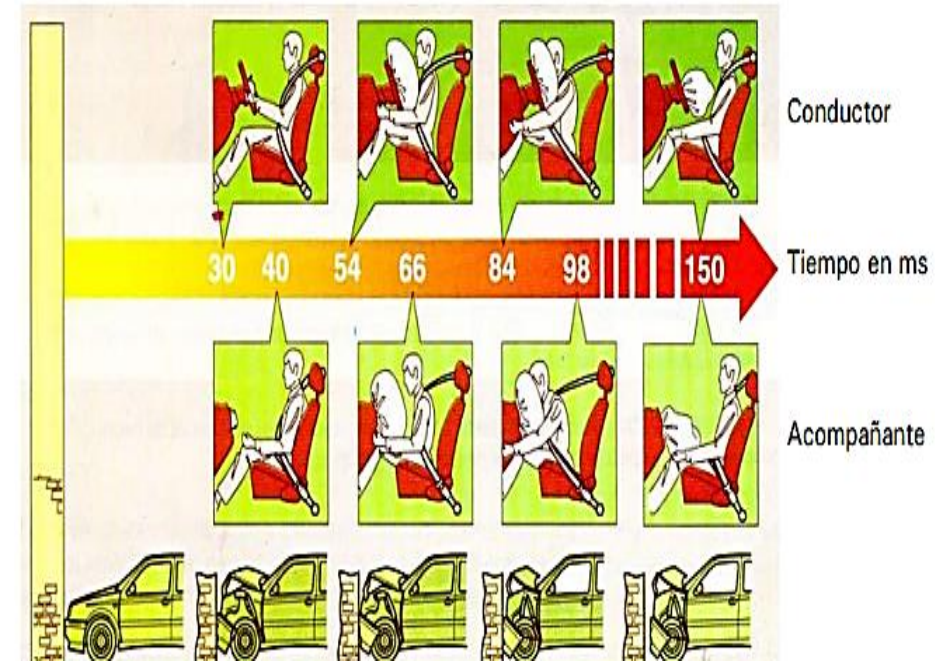

Figura 2: Proceso de expansión del cojín inflable del airbag del conductor y acompañante en relación al tiempo (milisegundos).

Fuente: Volkswagen; elementos electrónicos de seguridad.

\section{Resultados y Discusión}

Según El Futuro Del Sector Automotriz (2025) [3], estima que para dicho año todas las firmas automotrices equipen el sistema airbag independientemente del modelo o si se trata de un vehículo gama baja o alta el vehículo.

Luego procedemos a la visualización y estudio de campo en el vehículo que va a servir como banco de prueba.

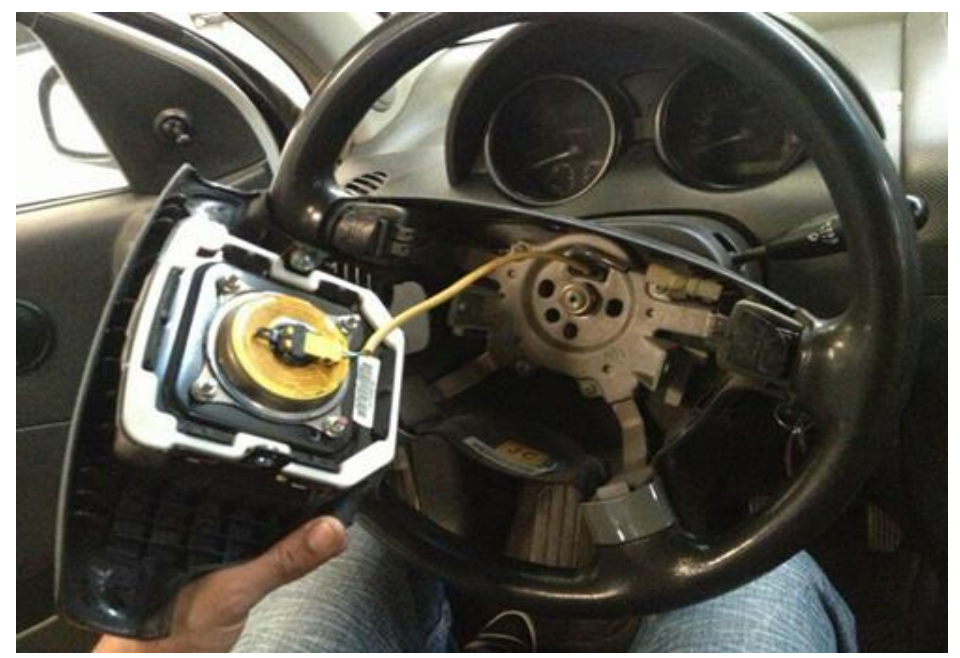

Figura 3: Localización del airbag del piloto en el vehículo Chevrolet Aveo 2008. 


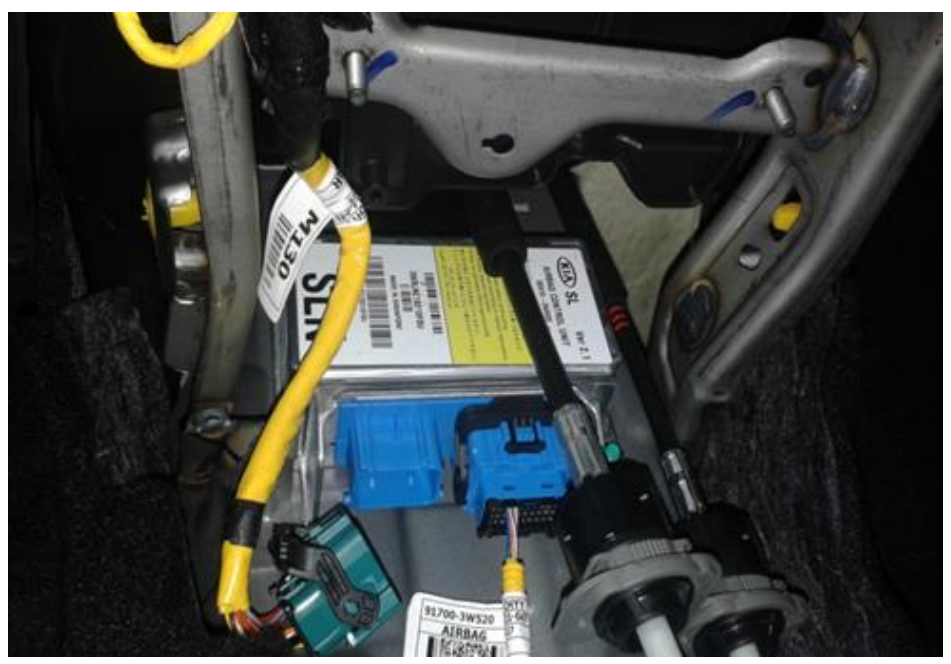

Figura 4: Programador de unidad de control.

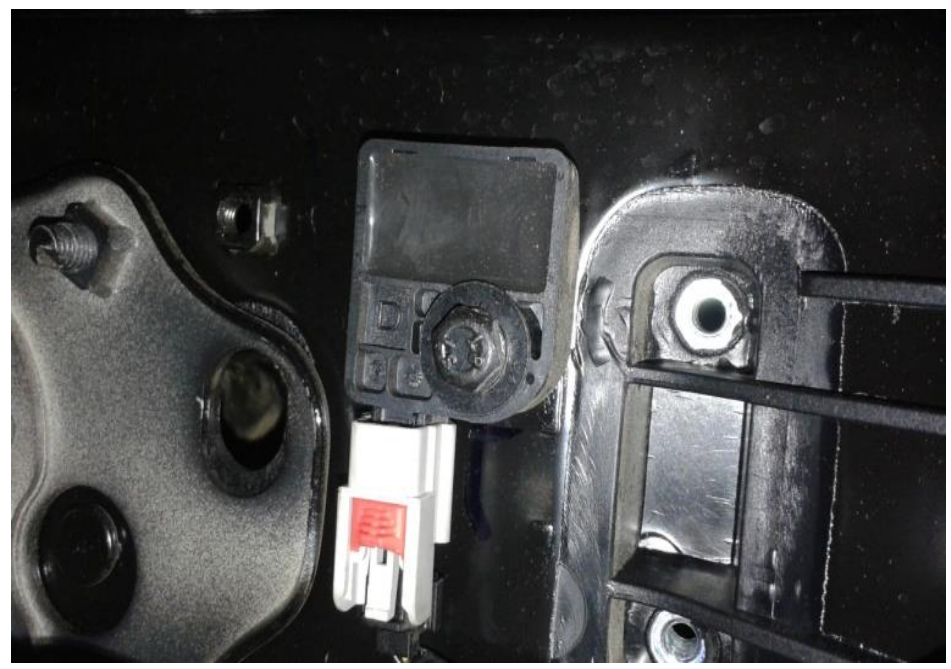

Figura 5: El sensor de deceleración del sistema.

Para el sitio web Actualidad motor [4] en la sección tipos de sensores de desaceleración hay tres tipos los cuales son: Mecánicos, Electrónicos, Electromagnéticos. En este caso nuestro sensor es electrónico. 


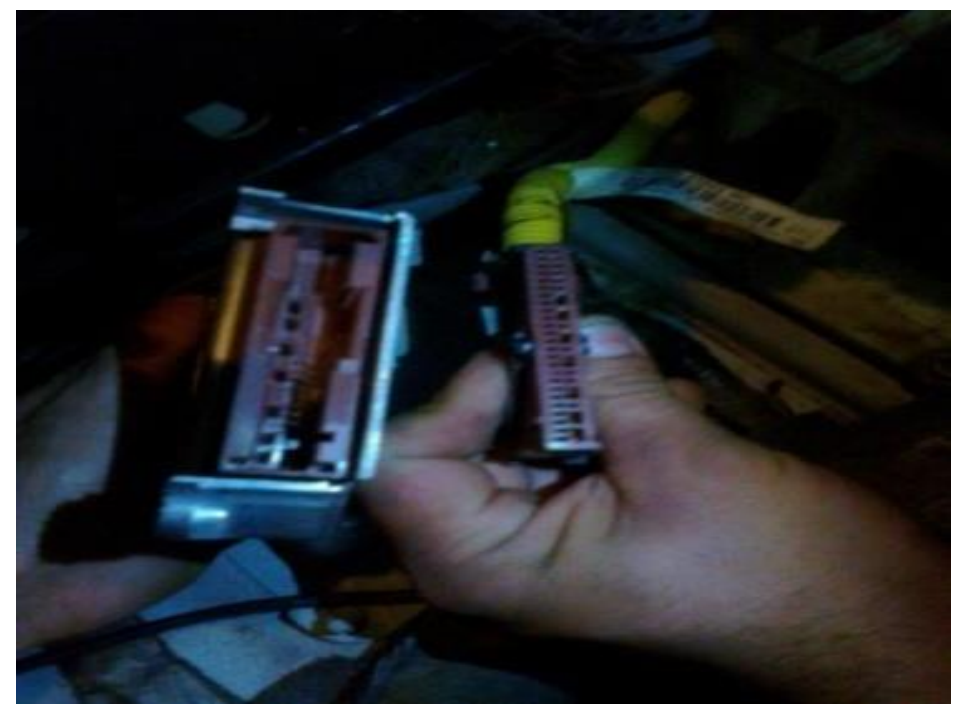

Figura 6: Los conectores de la Unidad de control.

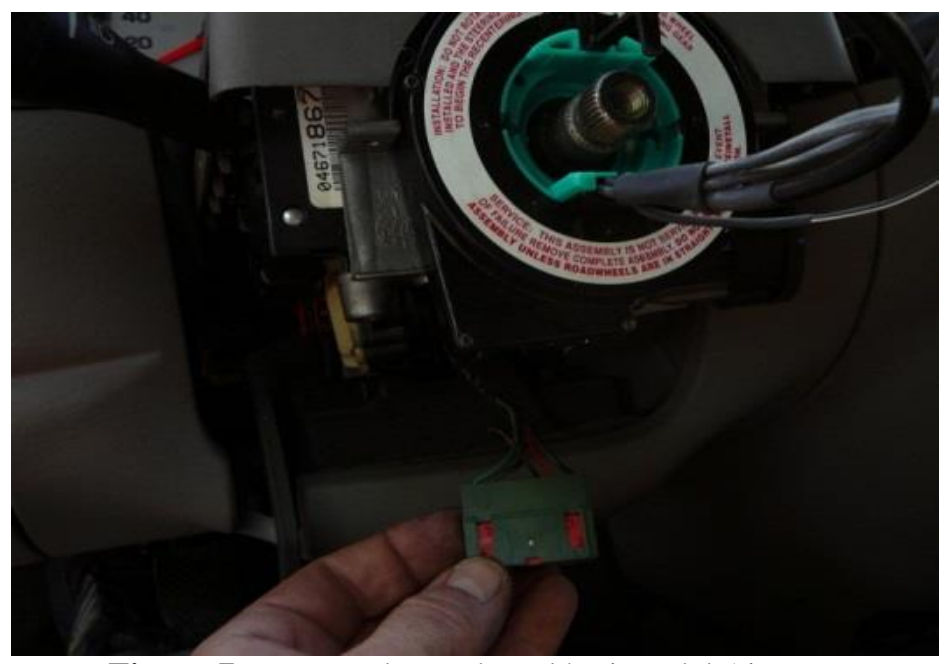

Figura 7: Proceso de pruebas eléctricas del Air Bag.

Posteriormente de realizar las identificación de componentes que conformar al airbag, su función y las pruebas de campo podemos asegurar que todas las tesis sobre seguridad pasiva tienen correlación la con este componente ya que la función que desempeña es muy importante y que las proyección que en el 2025 [3] se va a poder estandarizar en líneas de producción este sistema no está tana alejado de la realidad ya que se está convierto en un aliado importante para logar disminuir las tasas de mortalidad a nivel mundial, aunque hablando en nuestro país según $\underline{W O R L D}$ HEALTH RANKINGS 157 la tasa por accidentes de tránsito ECUADRO ocupa el puesto $17^{\circ}$ (diecisiete) entre 172 (ciento setenta y dos) países lo cual de cada 100.000 (cien mil) personas que sufren accidentes en nuestro país fallecen el 30,68\% lo cual nos dice que aproximadamente 30.680 (treinta mil seiscientos ochenta) personas fallecen cada cien mil personas y luego se distribuye entre peatones, conductores de motocicletas, ocupantes, conductores de vehículos y ocupantes. Pero es algo realmente grave porque en el tema automotor el $30 \%$ de esas 30.680 (treinta mil seiscientos ochenta) son solo muertes entre conductor y ocupantes, entonces el sistema airbag va en busca de la disminución de ese porcentaje y un $50 \%$ de los 30.680 se fija en peatones lo cual una de las marcas entre ellos Volkswagen [1] está desarrollando airbag para peatones. 
Con esta investigación se logró analizar y detectar las fallas más comunes que presenta el sistema airbag, que se centra principalmente en la muchas veces en el que sistema airbag no logra accionarse por problemas de señal del sensor o el ángulo de impacto lo que provoca el mal funcionamiento del sistema y por ende el riesgo fatal de lesiones graves de los ocupantes del vehículo, esto se pudo analizar mediante la toma de datos y pruebas realizadas con instrumentos de diagnóstico de alta tecnología al vehículo.

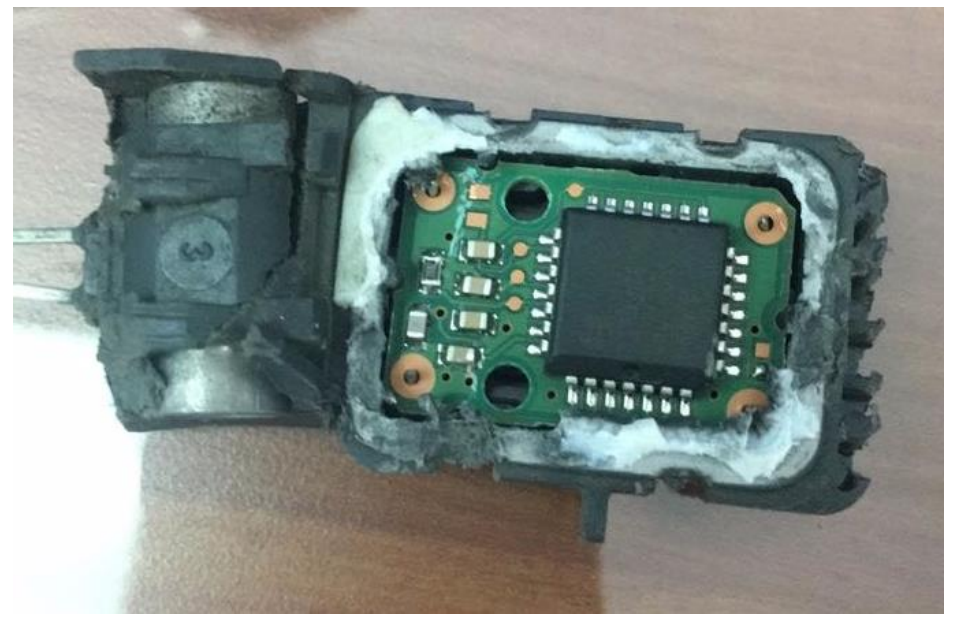

Figura 8: Sensor de impacto delantero sistema Air Bag.

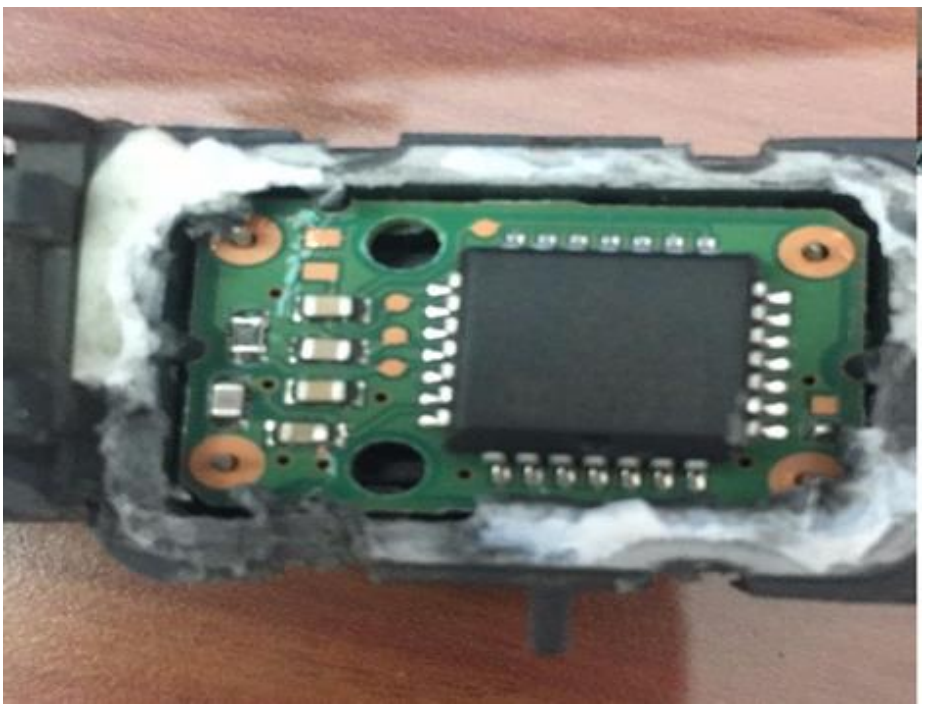

Figura 9: Sensor de impacto delantero sistema Air Bag. Circuito y visualización del microprocesador o memoria.

\section{FLUJOGRAMA DE OPERACIÓN.}




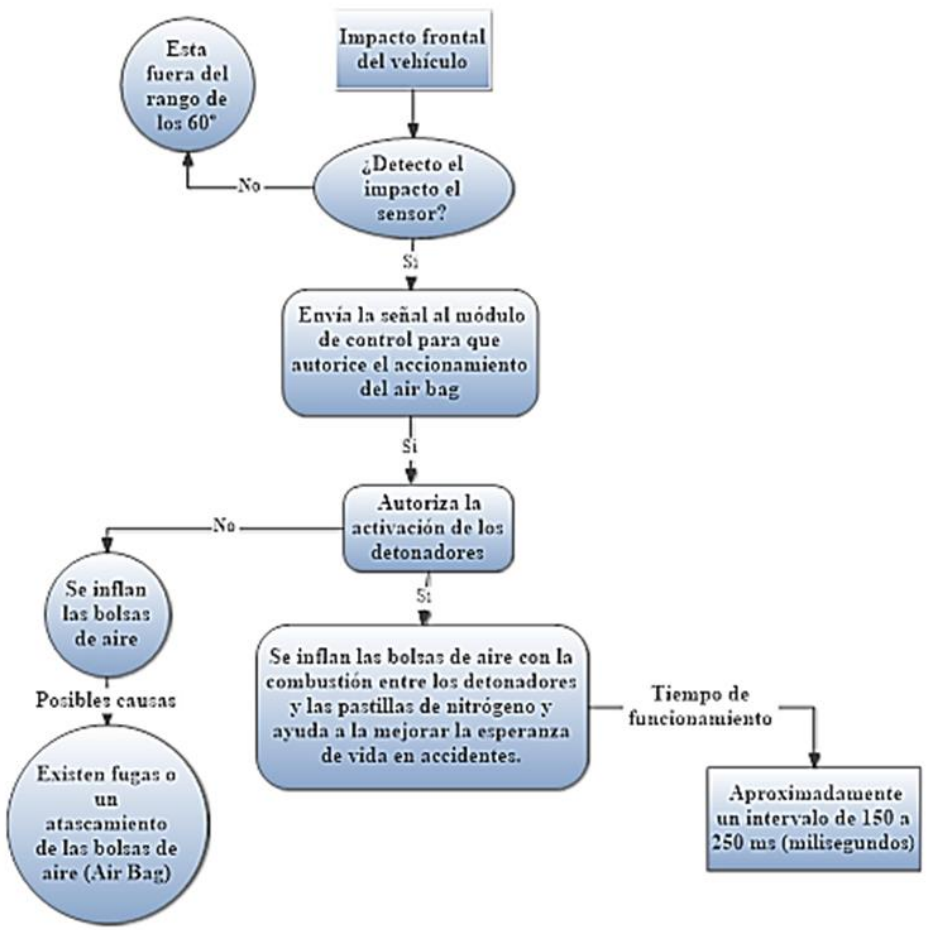

Figura 10: Flujograma de operación del sensor de impacto delantero, como se activa y causas de su no activación.

Software: SmartDraw.

\section{Conclusiones}

Se deben realizar las respectivas pruebas con el equipo de diagnóstico adecuado como multímetro automotriz, osciloscopio entre otros y con la ayuda de los manuales del fabricante, para saber si el sistema está trabajando dentro de los rangos admisibles y con el propósito de no averiar los elementos del sistema Airbag.

Se logró desarrollar flujo gramas de pruebas del sistema, con lo cual se pudo identificar de manera clara las fallas comunes del sistema Airbag, y así dar un diagnóstico preciso luego de evaluado el sistema.

Es importante la inclusión de la sociedad en la embarcación, capacitación y sobre sobre todo el pro y contra que posee el sistema airbag ya que al no estar concientizado sobre los problemas que puede acarrear que el sistema no se accione en el momento de una colisión (accidente) y si después de unos cuantos días en ese momento estaríamos frente a una bomba de tiempo.

El Ecuador debe fomentar y crear proyectos de leyes para que las importaciones, construcción y ensamble de vehículos ya vengan incorporado el sistema airbag y que los costos sean accesibles ante la real economía que tiene el país, para poder disminuir la tasa de mortalidad antes citada en el campo automotriz [5], ya que es transcendental incorporar este sistema de protección pasiva.

\section{OSCILOGRAMAS DE OPERACIÓN}




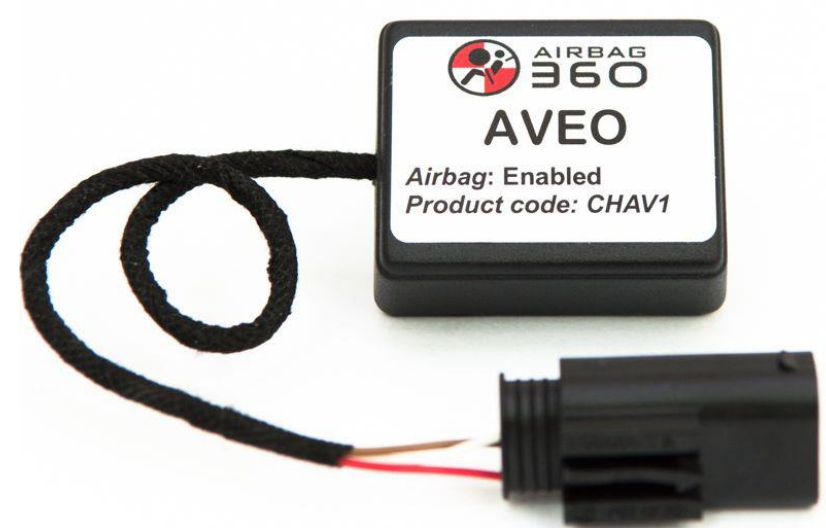

Figura 11: Sensor de impacto sistema Air Bag. Chevrolet Aveo. Fuente: http://airbag360.com/chevrolet-aveo-frontpassenger-side-airbag-seat--occupancy-sensor-b1448--emulator-187-p.asp

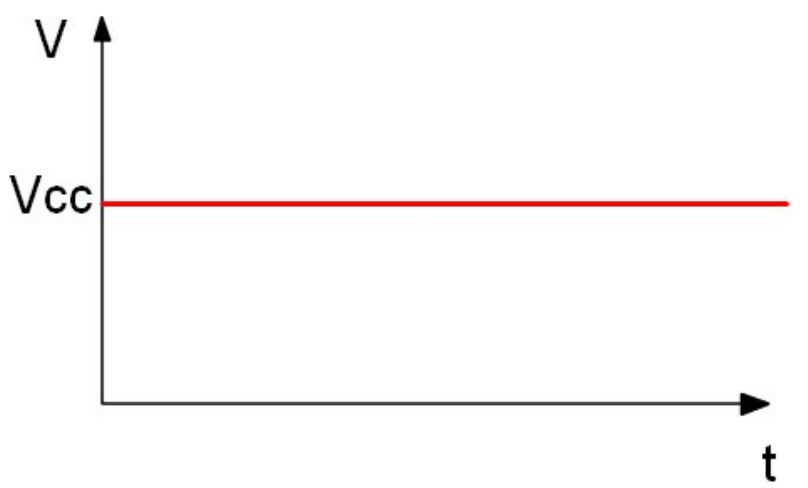

Figura 12: Grafica de voltaje contra tiempo del Sensor de impacto sistema Air Bag. Chevrolet Aveo.

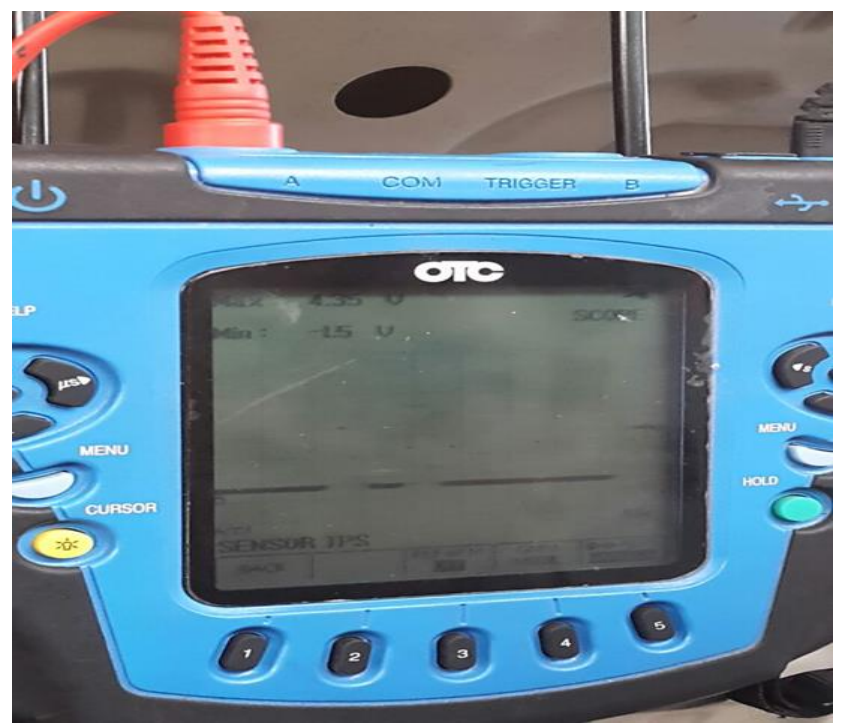

Figura 13: Grafica de voltaje contra tiempo del Sensor de impacto sistema Air Bag. Chevrolet Aveo. Obtenida en el osciloscopio. 
Se diseñó y elaboró el protocolo de pruebas para el sistema Airbag del vehículo, esto se lo realizó en base a los parámetros normales de operación y oscilogramas característicos de los elementos

El desarrollo del flujo gramas de pruebas, se deben realizar las respectivas pruebas con el equipo de diagnóstico adecuado y con la ayuda de los manuales del fabricante, para tener estudiados los parámetros de funcionamiento y con el propósito de no averiar los elementos del sistema Airbag, ya que sus costos de cambio más no de reparación son elevados para nuestro medio.

La forma de onda del sistema airbag en sus sensores son de efecto hall (onda cuadrada) o senoidal dependiendo del tipo de inducción que obtenga en nuestro caso es electrónico por lo cual la forma de onda es cuadrada y solo se obtiene la forma de onda simulando el impacto.

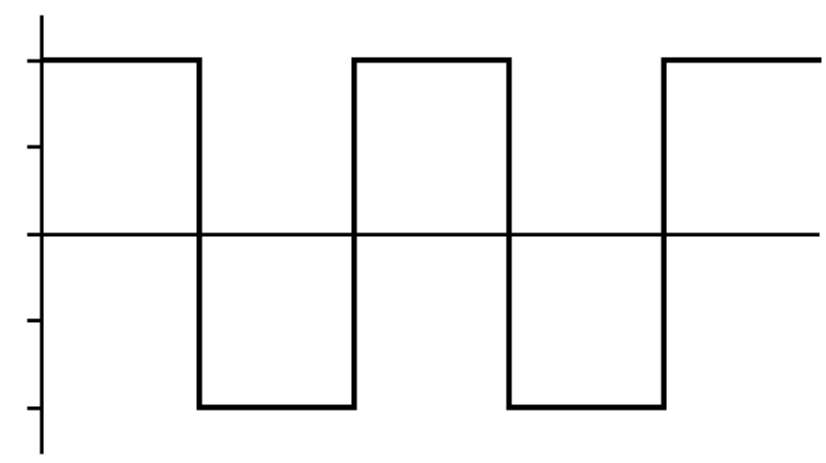

Figura 13: Grafica de voltaje contra tiempo del Sensor de impacto sistema Air Bag. Chevrolet Aveo. Simulando la colisión.

\section{Bibliografía}

Manual de Servicio Chevrolet Aveo español 2004-2008. Manual del propietario Chevrolet Aveo 2008. Software sistema de seguridad Air Bag Chevrolet Aveo 2008.

VOLKSWAGEN MEXICO INC, Elementos Electrónicos de Seguridad Descripción Técnica y Funcionamiento (2010)

Sica, D. Scarlan, M. Rossini, D. Beinstein, E. Figueroa, D. (2014). El Futuro Del Sector Automotriz En El Mundo (2025). Fuerzas impulsoras y tecnologías clave para su desarrollo en el marco de políticas que promuevan la calidad de vida y la conservación del medio ambiente y de los recursos naturales. Recuperado 12 de marzo de 2017, de http://www.mincyt.gob.ar/estudios/el-futuro-del-sector-automotriz-en-el-mundo-202510236

Taller virtual (2009). Tipos de sensores de desaceleración. Recuperado 10 de octubre de 2016, de https://www.actualidadmotor.com/tipos-de-sensores-de-desaceleracion. 
WORLD HEALTH RANKINGS (2016). Road traffic accidents, Death rate per 100,000. Recuperado 14 de marzo de 2017, de http://www.americaeconomia.com/politicasociedad/sociedad/revise-el-ranking-de-los-paises-con-mayores-tasas-de-muerte-poraccidente

Marcos Vallespí López (2016). El Airbag Recuperado 20 marzo 2017 www.ehu.es/zorrilla/juanma/automovil/airbag.htm

Revista CESVI (2016). Airbag y Cinturones de Seguridad, Unidos por la Seguridad. Recuperado el 22 de marzo 2017, de https://www.cesvi.com.ar/revistas/r74/airbags_74.pdf

José María Hernández Hernández (2007). El Airbag, Intervención y Rescate en accidentes de tráficos. $\quad$ Recuperado ttps://www.dipualba.es/sepei/pdfs/Documentaci\%C3\%B3n\%20\%20airbag.pdf

Santiago L. de Soria, Diciembre (2001). El Airbag un soplo de vida. Recuperado de http://www.centrozaragoza.com:8080/web/sala_prensa/revista_tecnica/hemeroteca/articulo s/R10_A7.pdf

Enrique ALCALÁ (INSIA-UPM Unidad de Investigación de Accidentes) febrero 2016. El Airbag: cómo funciona. Recuperado de http://revista.dgt.es/es/motor/tecnologiaseguridad/2016/0218-Airbags-como-son-como-funcionan.shtml\#.WNNYq1WGPIU

Chevrolet. Catálogo de repuesto. Recuperado 21 de marzo de 2017, en http://airbag360.com/chevrolet-aveo-front-passenger-side-airbag-seat--occupancy-sensorb1448--emulator-187-p.asp 